\title{
Application of inverse method to estimation of boundary conditions during investment casting simulation
}

\section{Haipeng JIN * and Jiarong LI}

National Key Laboratory of Advanced High Temperature Structural Materials, Institute of Aeronautical Materials, Beijing 100095, China

Dong PAN

Key Lab for Advanced Materials Processing Technology, MOE, Department of Mechanical Engineering, Tsinghua University, Beijing 100084, China

Manuscript received 6 January 2009; in revised form 20 March 2009

\begin{abstract}
Inverse method was used in single crystal superalloy DD6 processing simulation during solidification. Numerical modeling coupled with experiments has been used to estimate the interface heat transfer coefficient (IHTC) between the surface of slab casting and inner mold. Calculated temperature dependent values of IHTC were obtained from a numerical solution. The calculated temperatures agreed well with the measurement of cooling profile.

KEY WORDS Numerical simulation; Single crystal; Inverse method; Boundary conditions
\end{abstract}

\section{Introduction}

Many critical components in aerospace and other key industries are manufactured by the investment casting process ${ }^{[1]}$. Often these components are made of costly superalloys, are of complex shapes and vary greatly in size. Moreover, since investment casting itself is a complex process, the old-fashioned trial and error approach to selecting process parameters are both time consuming and expensive. As a result, there is much interest these days in finite element simulation of solidification in the investment casting process ${ }^{[2,3]}$. One of the main difficulties associated with numerical simulation of solidification processes is the lack of thermophysical properties and/or boundary conditions. Accurate boundary conditions data are difficult to obtain at high temperatures, especially for nickel-based superalloys. The interface heat transfer coefficient (IHTC) as a decisive factor for accurate simulation of the solidification process has been extensively studied in the past three decades. The interface heat transfer between the casting and the mold is a complicated process. Meanwhile, it plays an important role during radiation heat transfer processes. At present, three methods are applied to deal with this issue ${ }^{[4-10]}$.

\footnotetext{
${ }^{*}$ Corresponding author. Engineer, PhD; Tel: +86 10 62496335; Fax: +86 1062496371.

E-mail address: jhp9@yahoo.cn (Haipeng JIN)
}

DOI: $10.1016 / \mathrm{S} 1006-7191(08) 60119-2$ 
(1) Interface gap $h=\lambda_{\mathrm{g}} / x_{\mathrm{g}}+C\left(T_{1}^{2}+T_{2}^{2}\right)\left(T_{1}+T_{2}\right) /\left(1 / \varepsilon_{1}+1 / \varepsilon_{2}-1\right)$. Where, $\lambda_{\mathrm{g}}$ is the mean thermal conductivity of the gas, $x_{\mathrm{g}}$ is width of the gas, $C$ is Stefan-Boltzman constant, $T_{1}$ is the temperature at the outside of casting, $T_{2}$ is the temperature at the inner side of mold. $\varepsilon$ is emissivity of casting and mold. This method is not an effective manner to obtain interface coefficients due to the absence of $x_{\mathrm{g}}, T_{1}$ and $T_{2}$. (2) Inverse method. This method bases upon the experimental data to determine the interface coefficient. (3) Interface temperature difference. A lot of challenges remain to overcome for this method.

Using measurements made under well-defined conditions, numerical simulation can be used to deduce these unobtainable data. This is so-called inverse method, which is applied in the ProCAST. The basic idea of this method is equivalent to a standard least-squares method in which the analytical function is replaced by the numerical solution obtained from a direct finite element calculation. In this paper, boundary conditions were estimated using inverse method during directional solidification of single crystal superalloy DD6.

\section{Model Theories}

\subsection{Macro scale model}

A three-dimensional finite element solver, ProCAST (ESI Software, Paris), was applied to solve for the heat transfer during solidification at the macro scale. The model solves the heat transfer using the conservation of energy with temperature/time boundary conditions and temperature dependent material properties ${ }^{[11]}$ :

$$
\rho \frac{\delta H}{\delta T} \frac{\delta T}{\delta t}-\nabla[K \nabla T]-S(r)=0
$$

where $\rho$ is density, $T$ is temperature, $t$ is time, $K$ is thermal conductivity, $S(r)$ is the source term and $H$ is the enthalpy of solidification. Substituting $S(r)$ with the latent heat of fusion during solidification process, Eq.(1) can be rewritten as

$$
\rho C_{\mathrm{p}} \frac{\delta T}{\delta t}=\nabla[K \nabla T]+\rho L \frac{\delta f_{\mathrm{s}}}{\delta t}
$$

where $C_{\mathrm{p}}$ is the isobaric specific heat, $L$ is the latent heat of fusion and $f_{\mathrm{s}}$ is the fraction solid.

A radiation boundary condition was considered in the macrosacle model. The radiation was calculated using a view factor model: rather than tracking the reflected radiant energy from each element, an overall energy balance for each participating surface is considered ${ }^{[12]}$.

\subsection{Inverse model}

Consider a domain $\Omega$, within which the heat flow equation has to be solved. This domain includes the solidifying ingot and the mould parts. A set of $N_{\mathrm{m}}$ thermocouple has been placed at well defined positions $X_{j}\left(j=1, N_{\mathrm{m}}\right)$ within this domain in order to measure the temperature $T_{i j}^{\mathrm{m}}$ at a certain number of times $t_{i}\left(i=1, N_{\mathrm{t}}\right)$. These measured temperatures are used to deduce a set of $N_{\beta}$ parameters $\beta=\left\{\beta_{1}, \beta_{2} \cdots, \beta_{\mathrm{N} \beta}\right\}$ via a minimization of the function ${ }^{[13]}$.

$$
S(\beta)=\sum_{i=1}^{N_{\mathrm{t}}} \sum_{j=1}^{N_{\mathrm{m}}} \frac{1}{\sigma_{\mathrm{T}}^{2}}\left[T_{i j}^{\mathrm{m}}-T_{i j}^{\mathrm{c}}(\beta)\right]^{2}+\sum_{k=1}^{N_{\beta}} \frac{1}{\sigma_{\mathrm{k}}^{2}}\left[\beta_{\mathrm{k}}-\beta_{\mathrm{k}}^{0}\right]^{2}
$$


where $T_{i j}^{\mathrm{c}}(\beta)$ are the calculated temperature at time $t_{i}$ and position $X_{j}$. The standard deviation, $\sigma_{\mathrm{T}}$, is a typical error associated with the temperature measurement, whereas $\sigma_{\mathrm{k}}$ is a typical interval within which each of the parameter $\beta_{\mathrm{k}}$ is allowed to vary around a priori parameter $\beta_{\mathrm{k}}^{0}$. On the other hand, a parameter $\beta_{\mathrm{k}}$ will be fixed to the guessed value $\beta_{\mathrm{k}}^{0}$, if the corresponding deviation $\sigma_{\mathrm{k}}$ is made very small.

In order to minimize $S(\beta)$, Eq.(3) writes:

$$
\frac{\partial S}{\partial \beta_{1}}=\sum_{i=1}^{N_{\mathrm{t}}} \sum_{j=1}^{N_{\mathrm{m}}} \frac{-2}{\sigma_{\mathrm{T}}^{2}}\left[T_{i j}^{\mathrm{m}}-T_{i j}^{\mathrm{c}}(\beta)\right] \cdot X_{i j 1}+\frac{2}{\sigma_{1}^{2}}\left[\beta_{1}-\beta_{1}^{0}\right]=0
$$

where $X_{i j 1}$ is the sensitivity coefficient:

$$
X_{i j 1}=\frac{\partial T_{i j}(\beta)}{\partial \beta_{1}} \cong \frac{T_{i j}^{\mathrm{c}}\left(\beta_{1}, \cdots, \beta_{1}+\delta \beta_{1}, \cdots, \beta N_{\beta}\right)-T_{i j}^{\mathrm{c}}\left(\beta_{1}, \cdots, \beta_{1}, \cdots, \beta N_{\beta}\right)}{\delta \beta_{1}}
$$

where, $\delta \beta_{1}$ is a priori variation of the parameter $\beta_{1}$, which is used to calculate the sensitivity coefficients. An iterative procedure is used to find the solution $\beta$ minimising $S(\beta)$. In this procedure the calculated temperatures $T_{i j}^{\mathrm{c}}\left(\beta^{\nu+1}\right)$ at the next iteration $(\nu+1)$ are also linearized as

$$
T_{i j}^{\mathrm{c}}\left(\beta^{\nu+1}\right) \cong T_{i j}^{\mathrm{c}}\left(\beta^{\nu}\right)+\sum_{k=1}^{N_{\beta}} X_{i j \mathrm{k}} \cdot \Delta \beta_{\mathrm{k}}
$$

The increments $\Delta \beta$ of the parameter are then found at each iteration as the solution of the set of linear equations:

$$
[A] \cdot \Delta \beta=f \quad \text { or } \sum_{k=1}^{N_{\beta}} A_{1 \mathrm{k}} \cdot \Delta \beta_{\mathrm{k}}=f_{1}
$$

With:

$$
\begin{aligned}
A_{1 \mathrm{k}} & =\sum_{i=1}^{N_{\mathrm{t}}} \sum_{j=1}^{N_{\mathrm{m}}} \frac{X_{i j k} \cdot X_{i j l}}{\sigma_{\mathrm{T}}^{2}}+\frac{\delta_{1 \mathrm{~K}}}{\sigma_{1}^{2}} \\
f_{1} & =\sum_{i=1}^{N_{\mathrm{t}}} \sum_{j=1}^{N_{\mathrm{m}}} \frac{1}{\sigma_{\mathrm{T}}^{2}}\left[T_{i j}^{\mathrm{m}}-T_{i j}^{\mathrm{c}}\left(\beta^{\nu}\right)\right] \cdot X_{i j l}-\frac{\beta_{1}^{\nu}-\beta_{1}^{0}}{\sigma_{1}^{2}}
\end{aligned}
$$

$\delta_{1 \mathrm{~K}}$ is Kroneker symbol. The implementation of the inverse method (Eq.(7)) can be done in a direct program that calculates $T_{i j}^{\mathrm{c}}(\beta)$ once the parameters $\beta$ are known. From guessed initial values of $\beta^{(\nu=0)}$, the temperatures $T_{i j}^{\mathrm{c}}\left(\beta^{\nu}\right)$ and sensitivity coefficients $X_{i j l}$ (Eq.(5)) are calculated at each iteration $\nu$. Knowing these values, Eq.(7) are solved in order to obtain the increments $\Delta \beta$ until convergence is reached.

\section{Experimental}

Acquisition of accurate thermal data was an essential element of these experiments. Investment casting process was employed to manufacture the nickel-base single crystal superalloy DD6 slab in a vacuum induction furnace. The chemical composition (wt pct) 
of the alloy used in this study is $\mathrm{Cr} 4.3$, Co 9, W 8, Mo 2, Al 5.6, Nb 0.5, Ta 7.5, Re 2, Hf 0.1 and Ni balance.

Point A, Point $\mathrm{B}$ and Point $\mathrm{C}$ were instrumented with a thermocouple located at the mid-point of the casting. Three thermal couples were placed at the center of the slab and at 7,9 and $11 \mathrm{~cm}$ for the block starter bottom, as shown in Fig.1.

The temperatures of the points during the cooling processing were recorded by IMC multichannel data acquisition apparatus. A withdrawal rate of $4.5 \mathrm{~mm} / \mathrm{min}$, which is approximately a maximum achievable rate for commercial castings with similar crosssections, was selected from this experiment.

\section{Simulation}

The process of an inverse simulation using the ProCAST software involves a number of steps, during which the geometry model building, the material properties, the boundary conditions, and the initial conditions are specified.

Modeling activity continues to be based upon the 3-D solid model of the initial cluster configuration. The model was built as one sector of the three-piece cluster and includes the part with helix, starter and chill plate geometry. The geometries of the components were created individually using I-DEAS, a 3D CAD commercial software of UGS. The geometry is axisymetric and corresponds accurately to the geometry and dimensions of the furnace. Emissivities were applied to all free surface of the model. The soaking and withdrawal processes were modeled. The contrast and relation between direct modeling and inverse modeling are shown in Fig. $2^{[15]}$.

The measured temperature of point $\mathrm{B}$ was converted to interface file $\mathrm{a}^{*}$. TXT format file and imported to the ProCAST inverse module.

\section{Results and Discussion}

\subsection{Temperature dependent interface heat transfer coefficient estimation}

Using the measured cooling curves shown in Fig.3, an inverse calculation of five temperature-tabulated heat transfer coefficients was performed. The results of such a calculation are displayed in Fig.4 under a constant withdrawal rate.

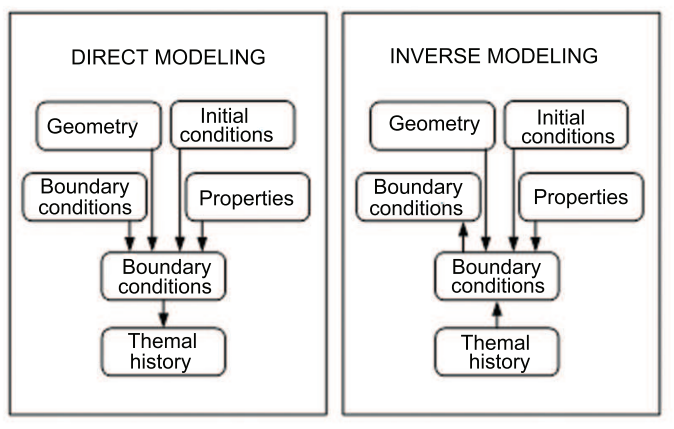

Fig.2 Principle of direct and inverse modeling ${ }^{[15]}$. 


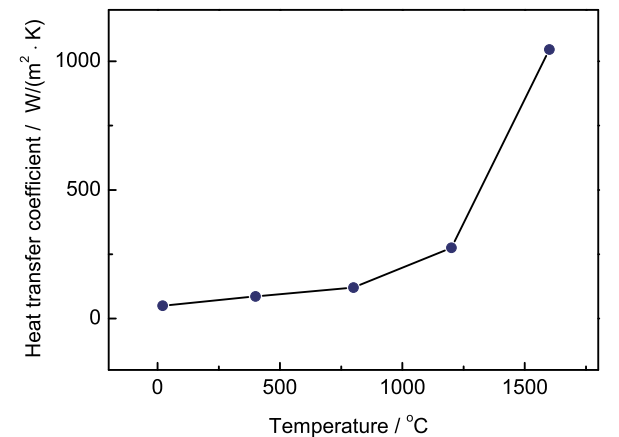

Fig.3 Temperature dependent heat transfer coefficient calculated by inverse modeling for a single crystal superalloy DD6 slab.

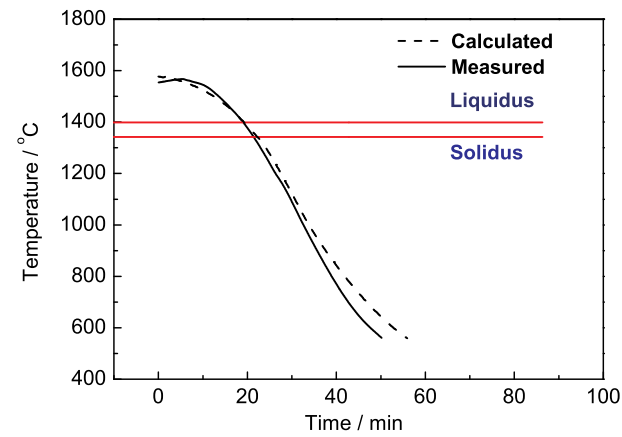

Fig.4 Comparison between thermocouple output data and predictions of model for thermal history at point B.

During these trials it was also necessary to vary the initial IHTC to maintain agreement with the measured cooling rate. These initial calculations yielded the nonconvergent results. Finally, the imposed temperatures were removed and further iterations performed with the new IHTC. The heat transfer coefficient is about $1000 \mathrm{~W} \cdot \mathrm{m}^{-2} \cdot \mathrm{K}^{-1}$ above the liquidus temperature. Below the solidus temperature, heat-transfer coefficient decayed exponentially from $500 \mathrm{~W} \cdot \mathrm{m}^{-2} \cdot \mathrm{K}^{-1}$ to almost zero at the room temperature. During experimental directional solidification, heat extraction is reduced below the solidus temperature by the formation of a gap between the cast part and the inner mold wall. Heat extraction switches to radiation when the solidification gap is formed, which results in the decrease of the heat transfer coefficient after the temperature variations ${ }^{[16]}$.

This can be assumed based on the fact that as the casting and mold cooled the temperature difference between the outer mold surface and the chamber walls decreased, which decreased radiation cooling from the outer mold surface (the primary method of heat extraction in the Bridgman process) and consequently the overall cooling rate and the temperature difference between materials ${ }^{[10]}$.

\subsection{Cooling curves}

Using ProCAST, the FEM solver, the thermal history of the casting was calculated. This included a fully $3 \mathrm{D}$ net radiation calculation with view factors. A comparison of the experimental and modeled thermal profiles, as shown in Fig.3, showed good agreement for temperatures in the solidification range of DD6 alloy.

\section{Conclusions}

(1) The present investigations have indicated that a direct FEM code coupled with thermal transfer can be used in an inverse method so as to calculate boundary conditions.

(2) A three dimensional inverse method has been used to obtain the interface heat transfer coefficient between the casting and the mold. The value of this heat transfer coefficient is temperature-dependent. Above the liquidus temperature, the value is about $1000 \mathrm{~W} \cdot \mathrm{m}^{-2} \cdot \mathrm{K}^{-1}$. Below the solidus temperature, heat-transfer coefficient decayed exponentially from $500 \mathrm{~W} \cdot \mathrm{m}^{-2} \cdot \mathrm{K}^{-1}$ to almost zero at the room temperature. 
(3) Using the boundary conditions, the solidification of single crystal superalloy DD6 slab casting has been simulated. Simulation results compare well with the experiments.

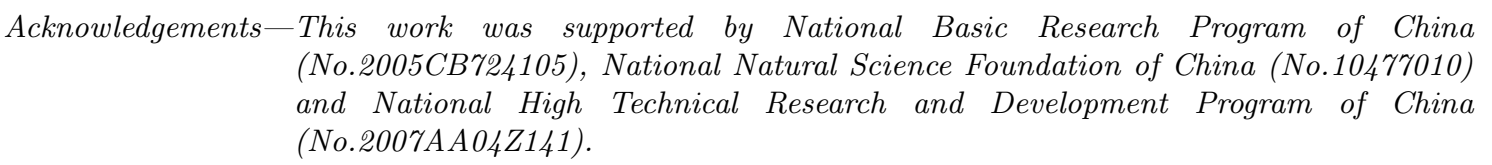

\section{REFERENCES}

[1] Casting: Metals Handbook. Vol.15, 9th ed., ASM International, Metals Park, Ohio, 1988.

[2] H.D. Brodv and D. Auelian, Modeling of Casting and Welding Processes (The Metallurgical Society of AIME, Pennsylvania, 1981).

[3] U. Chandra, Int J Numer Method Eng 30 (1990) 1301.

[4] P. Krishna, K.T. Bilkey, S.W. Hao and R.D. Pehlke, AFS Transaction (2004) p.71.

[5] S. Morimoto and A. Yoshinari, Superalloy 1988 (TMS, 1988) p.325.

[6] Philippe Bernard Lucien Auburtin. PhD Dissertation (The Columbia Univ, 1998).

[7] James William Stepanek. PhD Dissertation (The Carnegie Mellon Univ, 1998).

[8] Y.J. Kim, C.W. Lan and S. Kou, Proc 4th Int Conf On Modeling of Casting and Welding Process 1988.

[9] A.J. Elliott, PhD Dissertation (The Michigan University, 2005).

[10] X. Duan, Numerical Simulating and Calculating in Materials Science (Haerbin Institute of Technology Press, 2005) p.68 (in Chinese).

[11] R.B. Bird, W.E. Stewart and E.N. Lightfoot, Transport Phenomena Singapore (John Wiley \& Sons, Inc., 1960)

[12] ProCAST User's Manual \& Technical Reference. UES Software, Inc., 1998.

[13] G. Milano and F. Dcarpa, Private Communication.

[14] J.R. Li, Z.G. Zhong, D.Z. Tang, S.Z. Liu, P. Wei, P.Y. Wei, Z.T. Wu, D. Huang and M. Han, In: T.M. Pollock, R.D. Kissinger, R.R. Bowman, K.A. Green, M. McLean, S. Olson and J.J. Schirra eds., Superalloys 2000 (Warrendale, PA: TMS, 2000)

[15] ProCAST User Manual Version 2004.0, ESI Group, Paris, 2004.

[16] M. Konter, E. Kats and N. Hofmam, In: T.M. Pollock, R.D. Kissinger, R.D. Bowman, K.A. Green, M. McLean, S. Olson and J.J. Schirra eds., Superalloys 2000 (Warrendale, PA: TMS, 2000). 